\title{
The expression and evolution of virulence in multiple infections: the role of specificity, relative virulence and relative dose
}

\author{
Frida Ben-Ami ${ }^{*}$ and Jarkko Routtu
}

\begin{abstract}
Background: Multiple infections of the same host by different strains of the same microparasite species are believed to play a crucial role during the evolution of parasite virulence. We investigated the role of specificity, relative virulence and relative dose in determining the competitive outcome of multiple infections in the Daphnia magna-Pasteuria ramosa host-parasite system.

Results: We found that infections by P. ramosa clones (single genotype) were less virulent and produced more spores than infections by $P$. ramosa isolates (possibly containing multiple genotypes). We also found that two similarly virulent isolates of $P$. ramosa differed considerably in their within-host competitiveness and their effects on host offspring production when faced with coinfecting P. ramosa isolates and clones. Although the relative virulence of a P. ramosa isolate/clone appears to be a good indicator of its competitiveness during multiple infections, the relative dose may alter the competitive outcome. Moreover, spore counts on day 20 post-infection indicate that the competitive outcome is largely decided early in the parasite's growth phase, possibly mediated by direct interference or apparent competition.
\end{abstract}

Conclusions: Our results emphasize the importance of epidemiology as well as of various parasite traits in determining the outcome of within-host competition. Incorporating realistic epidemiological and ecological conditions when testing theoretical models of multiple infections, as well as using a wider range of host and parasite genotypes, will enable us to better understand the course of virulence evolution.

\section{Background}

In nature, free-living organisms are regularly found to be infected by an assemblage of different parasite species or genetically distinct parasite strains (reviewed in $[1,2]$ ). In fact, multiple infections are the norm rather than the exception in diverse host-parasite systems, e.g., anthersmut disease [3], malaria Plasmodium spp. [4], insect nucleopolyhedrovirus [5], fungus gardens of the leafcutter ants [6]. Coinfections also have implications for human health [7]. Theory has emphasized the importance of multiple infections in a variety of evolutionary processes such as the emergence of resistance to drugs [8], the evolution of sex in hosts coevolving with multiple parasites [9] and particularly the evolution of higher levels of virulence [10-13]. In the absence of spiteful

\footnotetext{
*Correspondence: frida@post.tau.ac.il

* Correspondence: frida@post.tau.ac.il University, Tel Aviv 6997801, Israel
}

(c) 2013 Ben-Ami and Routtu; licensee BioMed Central Ltd. This is an Open Access article distributed under the terms of the

interactions among parasite strains [14] or when the reproductive or exploitative rate of an individual parasite is not limited by the collective action of the coinfecting group [15], most experimental studies suggest that the overall expression of virulence of multiple infections is either higher than the virulence of any of the coinfecting strains as measured in single infections [16-18], or at least as high as the most virulent strain [19-22]. Understanding the determinants of intra-host competition and predicting the course of virulence evolution are thus of outmost importance for public health, medicine and agriculture [23].

The competitive outcome of multiple infections appears to be driven by several interrelated factors: the relative virulence of the coinfecting strains as measured in single infections [19,24], prior residency of one of the parasite strains or species [25-28], and the infectious dose used during simultaneous exposure $[18,29,30]$. The latter fac- 
tor - infectious dose - is strongly tied to the parasites' epidemiology. For example, variation in the infectious dose (i.e., the number of parasite spores a host is challenged with) is known to affect the probability of infection in single [31-33] and multiple infections [34]. Furthermore, the rate at which transmission stages are produced within the host could be influenced by the relative dose of its coinfecting parasites $[35,36]$. This is important, because all else being equal, a parasite strain that produces more transmission stages will have a greater representation in subsequent infectious doses. Since virulence is proposed to be traded off against parasite transmission ([37,38], reviewed in [39]), it is crucial to understand how the relative dose of each of the coinfecting parasite strains can impact this trade-off. Nevertheless, few studies examined how the relative parasite dose influences the expression of virulence and the production of transmission stages in the presence of multiple infections $[18,30]$.

Another often overlooked factor that may affect the expression and evolution of virulence is the degree of specificity in host-parasite interactions. In theory, highly specialized parasites can evolve towards high levels of virulence ([40], but see also [41]). For instance, peak parasitaemia (a proxy for virulence) was higher in specialist than in generalist malaria parasites of primates, when confounding life-history traits were controlled [42]. An earlier study of simultaneous and sequential multiple infections of Daphnia magna using three isolates of its obligate parasite Pasteuria ramosa suggested that the most virulent competitor produced most transmission stages [19]. However, P. ramosa clones (including the two clones used in the present study) have recently been shown to exhibit much higher specificity than isolates (clones are a single genotype whereas isolates are parasite samples from infected hosts that may contain multiple genotypes; [43]). In other words, $P$. ramosa clones infect fewer D. magna genotypes than $P$. ramosa isolates, and therefore the host genotype range of $P$. ramosa clones is narrower than that of isolates [43].

The present study experimentally investigates multiple infections in D. magna using infectious doses containing isolates and clones of P. ramosa in equal (50:50) and unequal proportions (90:10 and 10:90). By varying the relative representation of $P$. ramosa isolates/clones in the infectious dose (i.e., varying the specificity of multiple infections) and by comparing virulence, host fitness and parasite fitness in single vs. mixed infection treatments, we aim at (i) exploring how the relative virulence in single infections affects the overall expression of virulence during mixed infections, and (ii) assessing the effects of specificity ( $P$. ramosa isolates vs. clones) on intra-host competition. In the following we use the term single infections to refer to infectious doses containing either a single $P$. ramosa clone or a single $P$. ramosa isolate. We use the term mixed infections to refer to infectious doses containing a mixture of a single $P$. ramosa clone and a single $P$. ramosa isolate, or a mixture of two different $P$. ramosa isolates.

\section{Results}

\section{General effects}

Between days 5 and 16 of the experiment, 178 of 1,344 D. magna individuals died for unknown reasons (13.2\%). Such rates of early host deaths are not unusual $[19,27]$. None of the control D. magna became infected. Controls were excluded from the analyses of infection rates and parasite spore production. Infection rates in all infection treatments were above $90 \%$. The few uninfected D. magna in the infection treatments were excluded from all analyses. Time-to-host-death in the control group was on average $( \pm$ SE) twofold longer than that of all infection treatments combined $(112.7 \pm 3.7$ vs. $54.9 \pm 0.4$ days, Table 1[A]). Host control animals produced $251.3 \pm 8.3$ offspring per individual, whereas the average of the pooled infection treatments resulted in only $3.6 \pm 0.2$ offspring per individual.

\section{Virulence, host fitness and parasite fitness in single infections}

We found a significant difference in virulence (defined as time-to-host-death-since-exposure; see Methods further below) among the five parasite isolates/clones, with isolates $\mathrm{P} 1$ and $\mathrm{P} 2$ being the most virulent irrespective of the infective dose used (Table 2, Figure 1). Pasteuria ramosa isolates killed their host faster than $P$. ramosa clones (isolates vs. clones: $F_{1,464}=199.6, P<0.001$ ), regardless of dose (isolate/clone * dose: $F_{3,464}=0.3$, $P=0.85)$. Host offspring production was on average higher in hosts infected by isolate P1 than in hosts infected by the remaining isolates/clones, but it was invariant to dose (Table 2, Figure 2). Spore production differed among the five parasite isolates/clones, but was unaffected by dose (Table 2). Pasteuria ramosa isolates produced fewer spores than $P$. ramosa clones, regardless of dose (isolates vs. clones: $F_{1,464}=43.3, P<0.001$; isolate/clone $*$ dose: $\left.F_{3,464}=0.7, P=0.53\right)$. The results are similar with and without including the interactions in the model. Since dose level only slightly increased virulence, and even then without interacting with parasite isolate/ clone, in the following we only compared the higher dose, single infection treatments (100,000 spores) with multiple infections consisting of spore mixtures where the total amount of spores is 100,000 (i.e., 90,000:10,000, 50,000:50,000 and 10,000:90,000 spores).

\section{Virulence in mixed infections}

In the case of mixed infections with parasite isolate P1, time-to-host-death was unaffected by the relative 
Table 1 Infection contrasts

\begin{tabular}{|c|c|c|c|}
\hline \multirow[b]{2}{*}{ Source } & \multirow[b]{2}{*}{$d f$} & \multicolumn{2}{|c|}{$\begin{array}{l}\text { Time-to-host-death } \\
\text { (virulence) }\end{array}$} \\
\hline & & $F$ & $P$ \\
\hline Corrected model & 23 & 40.7 & $<0.001$ \\
\hline A. Control vs. infection treatments & 1 & 797.2 & $<0.001$ \\
\hline \multicolumn{4}{|l|}{ Infection contrasts } \\
\hline B. Mixed infections with P1 and P4 & 2 & 0.4 & 0.68 \\
\hline $\begin{array}{l}\text { C: P4-100 vs. P1-10 + P4-90 \& P1-50 + } \\
\text { P4-50 \& P1-90 + P4-10 \& P1-100 }\end{array}$ & 1 & 7.6 & $=0.006$ \\
\hline D: Mixed infections with P1 and C1 & 2 & 0.1 & 0.94 \\
\hline $\begin{array}{l}\text { E: C1-100 vs. P1-10 + C1-90 \& P1-50 + } \\
\text { C1-50 \& P1-90 + C1-10 \& P1-100 }\end{array}$ & 1 & 34.7 & $<0.001$ \\
\hline F: Mixed infections with P1 and C14 & 2 & 0.5 & 0.62 \\
\hline $\begin{array}{l}\text { G: C14-100 vs. P1-10 + C14-90 \& P1-50 + } \\
\text { C14-50 \& P1-90 + C14-10 \& P1-100 }\end{array}$ & 1 & 39.9 & $<0.001$ \\
\hline $\mathrm{H}$ : Mixed infections with P2 and P4 & 2 & 1.8 & 0.16 \\
\hline $\begin{array}{l}\text { I: P4-100 vs. P2-10 + P4-90 \& P2-50 + } \\
\text { P4-50 \& P2-90 + P4-10 \& P2-100 }\end{array}$ & 1 & 6.7 & $=0.01$ \\
\hline J: Mixed infections with P2 and C1 & 2 & 15.6 & $<0.001$ \\
\hline $\begin{array}{l}\text { K: C1-100 vs. P2-10 + C1-90 \& P2-50+ } \\
\text { C1-50 \& P2-90 + C1-10 \& P2-100 }\end{array}$ & 1 & 27.8 & $<0.001$ \\
\hline L: Mixed infections with P2 and C14 & 2 & 6.2 & $=0.002$ \\
\hline $\begin{array}{l}\text { M: C14-100 vs. P2-10 + C14-90 \& P2-50 + } \\
\text { C14-50 \& P2-90 + C14-10 \& P2-100 }\end{array}$ & 1 & 28.7 & $<0.001$ \\
\hline Error & 582 & & \\
\hline
\end{tabular}

ANOVA contrasts for time-to-host-death. $F$ statistic and significance in contrasts in which the degrees of freedom are greater than one are for the joint contrast. All contrasts are orthogonal. Bold typeface indicates significant effects.

proportions of the other isolate or clone (Table $1[\mathrm{~B} / \mathrm{D} / \mathrm{F}]$ ). Mixed infections with $\mathrm{P} 1$ were as virulent as single infections with P1 (Table $1[\mathrm{C} / \mathrm{E} / \mathrm{G}]$, Figure $1 \mathrm{~A}-\mathrm{C}$ ). In the case of mixed infections with parasite isolate P2, time-to-hostdeath was only unaffected by the relative proportions of parasite isolate $\mathrm{P} 4$ (Table $1[\mathrm{H}]$ ). In this case mixed infections with P2 were as virulent as single infections with P2 (Table 1[I], Figure 1D). Although P2 was as virulent as P1 in single infections ( $49.2 \pm 3.4$ vs. $48.9 \pm 1.8$ days), mixed infections with low concentrations of P2 (10\%) and high concentrations of parasite clones C1 or C14 (90\%) were as virulent as single infections with $\mathrm{C} 1$ or $\mathrm{C} 14$, respectively. Mixed infections with higher concentrations of P2 (50\% or $90 \%)$ were as virulent as single infections with P2 (Table $1[\mathrm{~J} / \mathrm{K} / \mathrm{L} / \mathrm{M}]$, Figure $1 \mathrm{E}-\mathrm{F}$ ).

\section{Host fitness in mixed infections}

In the case of mixed infections with parasite isolate P1, host offspring production tended to decline with increasing concentrations of P4 and C1 (Figure 2A-B), though this decline was not significant. Mixed infections with P1 and C14 in unequal concentrations (10:90 and 90:10) resulted in fewer offspring than single infections, but in equal concentrations (50:50) they resulted in more offspring than single infections $\left(F_{2,582}=5.8, P=0.003\right.$; Figure $\left.2 \mathrm{C}\right)$. In the case of parasite isolate $\mathrm{P} 2$, host offspring production did not differ among mixed infection treatments with P4, C1 or C14 $(P>0.97$; Figure 2D-F). Overall, mixed infection treatments with $\mathrm{P} 1$ resulted in the production of significantly more host offspring than with P2 $\left(F_{1,582}=8.7\right.$, $P=0.003$; Figure 2).

\section{Competitive outcome on day 20 post-infection}

We used genetic markers to test for the relative success of the competing parasite isolates and clones within individual hosts during the growth phase (day 20) of the disease and upon host death. The superior competitiveness of parasite isolate $\mathrm{P} 1$ in comparison with $\mathrm{P} 4, \mathrm{C} 1$ and $\mathrm{C} 14$ was largely evident on day 20 post-infection (Figure 3 ). More precisely, by day 20 isolate $\mathrm{P} 1$ produced more spores consistently and increasingly by relative dose: $1.43-$ 1.61 million more spores in concentrations of $10 \%, 1.93-$ 2.57 million more spores in concentrations of $50 \%$, and 2.27-3.13 million more spores in concentrations of $90 \%$ (P1 and P4: $\mathrm{F}_{2,27}=10.7, P<0.001 ; \mathrm{P} 1$ and $\mathrm{C} 1: \mathrm{F}_{2,25}=5.0$, $P=0.014 ; \mathrm{P} 1$ and $\mathrm{C} 14: \mathrm{F}_{2,27}=3.5, P=0.043$; Figure 3 ).

\section{Terminal competitive outcome}

Mixed infections with parasite isolate P1 in concentrations of $90 \%$ or $50 \%$ resulted in an almost complete exclusion of P4, C1 and C14 (95\% confidence interval for the difference in spore production between P1 and P4/ $\mathrm{C} 1 / \mathrm{C} 14$ did not include 0; Figure 4A-C). Only when P1

Table 2 Analysis of variance for host and parasite traits in single infection treatments

\begin{tabular}{|c|c|c|c|c|c|c|c|}
\hline \multirow[b]{2}{*}{ Source } & \multirow[b]{2}{*}{$d f$} & \multicolumn{2}{|c|}{$\begin{array}{l}\text { Time-to-host-death } \\
\text { (virulence) }\end{array}$} & \multicolumn{2}{|c|}{$\begin{array}{l}\text { Offspring production } \\
\text { (host fitness) }\end{array}$} & \multicolumn{2}{|c|}{$\begin{array}{l}\text { Spore production } \\
\text { (parasite fitness) }\end{array}$} \\
\hline & & $F$ & $P$ & $F$ & $P$ & $F$ & $P$ \\
\hline Parasite & 4 & 49.3 & $<0.001$ & 13.5 & $<0.001$ & 12.8 & $<0.001$ \\
\hline Dose & 3 & 5.0 & 0.02 & 2.0 & 0.16 & 2.8 & 0.08 \\
\hline Parasite * Dose & 12 & 1.2 & 0.27 & 0.5 & 0.91 & 0.9 & 0.52 \\
\hline Error & 452 & & & & & & \\
\hline
\end{tabular}

Two-way ANOVA for time-to-host-death, host offspring production and parasite spore production in single infection treatments. Bold typeface indicates significant effects. 


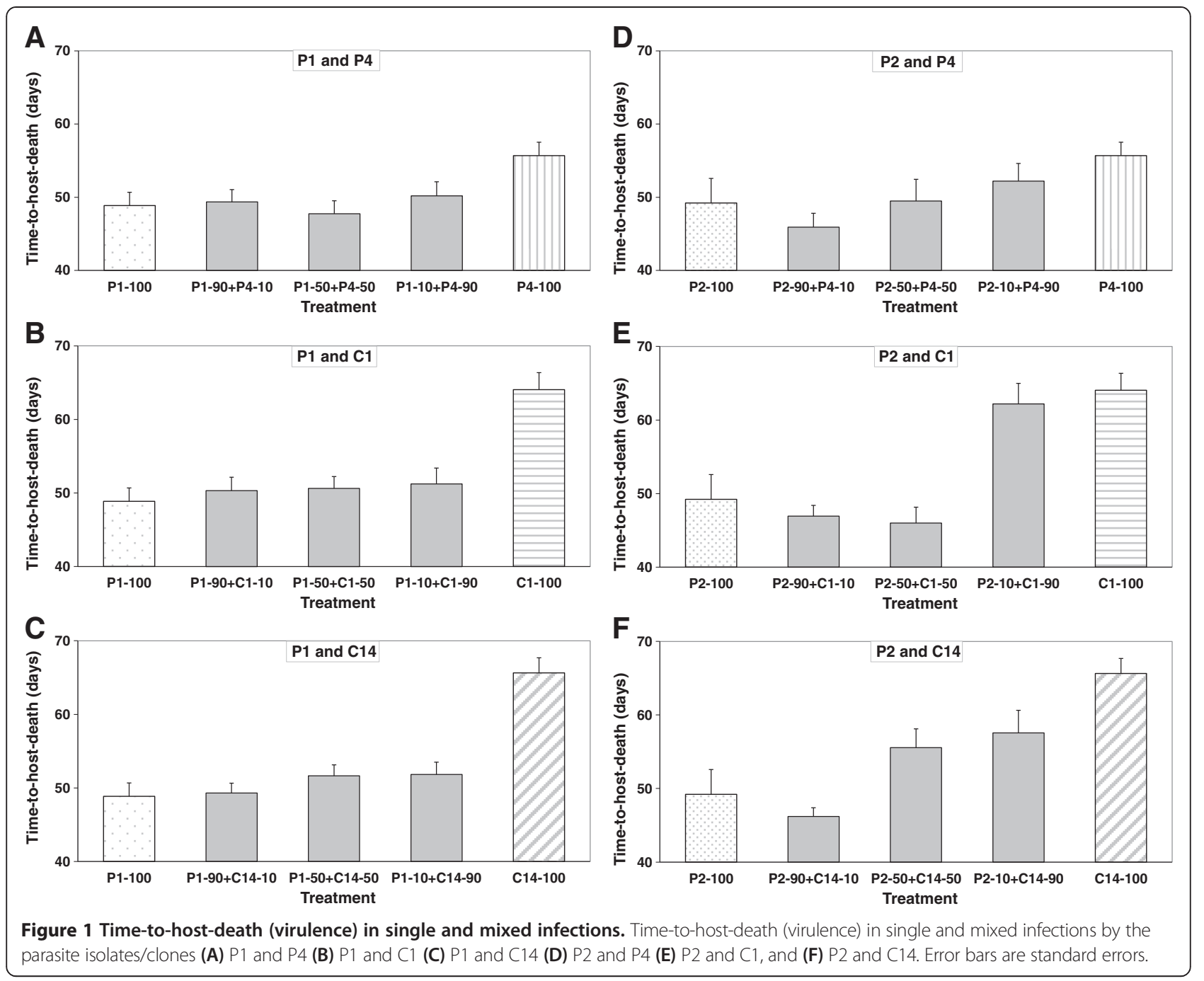

was present in a low starting concentration (10\%), both parasites succeeded in producing spores, but both suffered by producing fewer transmission stages than they produced in single infections (Figure 4A-C). In these low concentration treatments, spore production by isolate $\mathrm{P} 1$ exceeded that of $\mathrm{P} 4 / \mathrm{C} 1 / \mathrm{C} 14$, but the difference was not significant.

Although parasite isolate P2 was as virulent as P1 in single infections, in mixed infections it was less competitive than P1. Only when P2 started with 90\%, it mostly excluded the other strains (95\% confidence interval for the difference in spore production between P2 and P4/ $\mathrm{C} 1 / \mathrm{C} 14$ did not include 0; Figure 4D-F). In lower concentrations (i.e., $50 \%$ or $10 \%$ ) isolate P4 nearly excluded P2 (Figure 4D), whereas in the case of P2 vs. C1 or C14, each pair succeeded in producing spores, but both suffered by producing fewer transmission stages than they produced in single infections (Figure 4E-F).

Total spore production (i.e., sum of spores produced by a pair of parasites in mixed infections) did not significantly exceed the amount of spores produced by the parasite that produced more spores in the pair during single infections (Figure 4A-F).

\section{Discussion}

In single infections we found that $P$. ramosa isolates killed their hosts faster and produced fewer spores than $P$. ramosa clones. We also found that two similarly virulent isolates of $P$. ramosa differ considerably in their competitiveness when faced with coinfecting $P$. ramosa isolates and clones. While isolate P1 almost completely prevented the less virulent isolate $\mathrm{P} 4$ and the less virulent clones $\mathrm{C} 1$ and $\mathrm{C} 14$ from producing spores regardless of their relative dose (Figure 4A-C), in the case of isolate P2 the relative dose affected the competitive outcome (Figure 4D-F). Despite P1 being a better competitor, single and mixed infections with P1 resulted in the production of more host offspring than with P2 (Figure 2). Mixed infections were as virulent but not more virulent than single clone infections, and thus neither resulted in overexploitation of the host by 


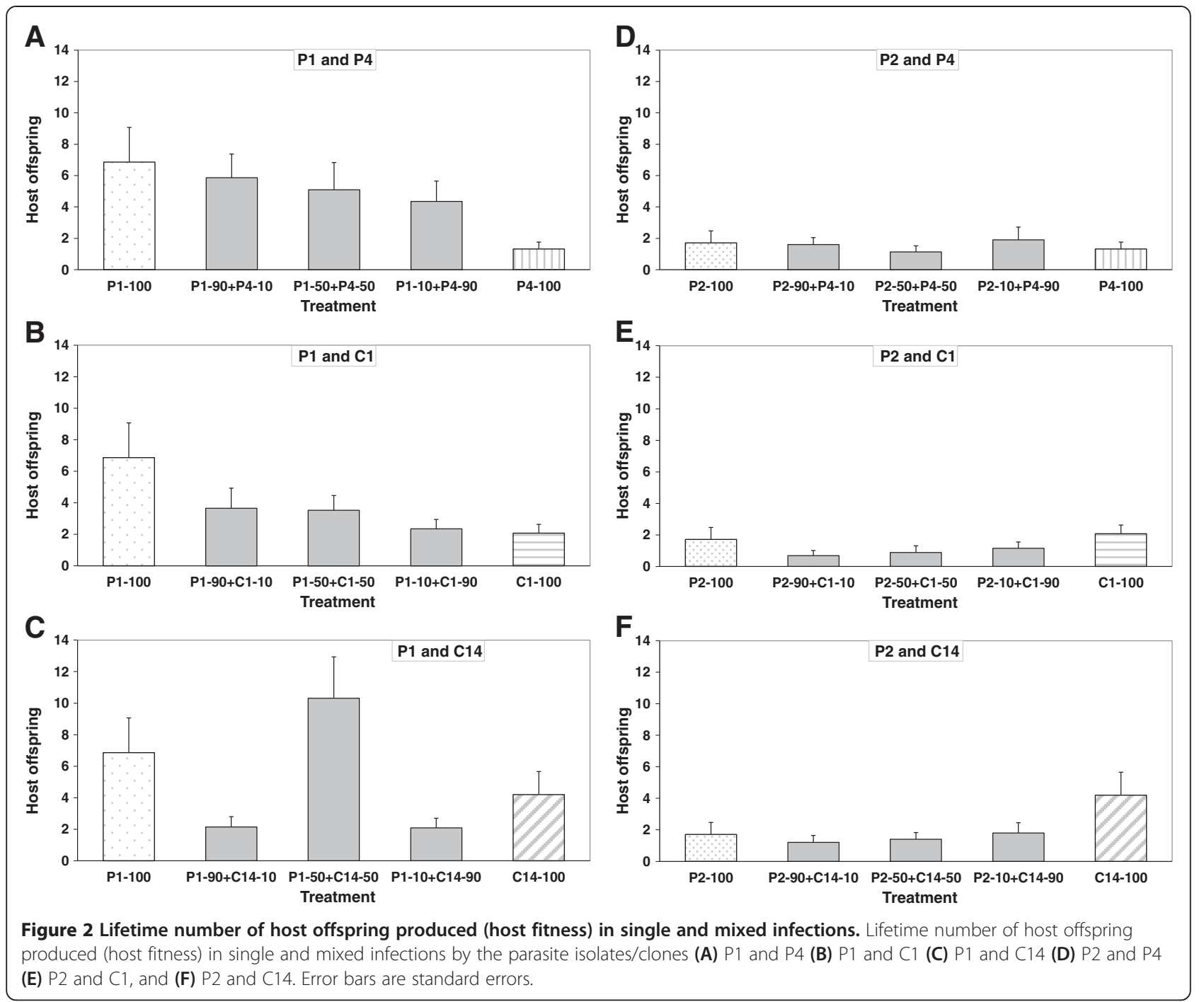

the parasites (i.e., time-to-host-death and total spore production were not higher in mixed infections; Figures 1 and 4), nor entailed additional costs upon the host because there was no further reduction in host fecundity (Figure 2). The competitive ability in treatments with equal concentrations (50:50) appears to be transitive, i.e., against both reference isolates $\mathrm{P} 1$ and $\mathrm{P} 2$, isolate $\mathrm{P} 4$ competes better than clone $\mathrm{C} 1$ which competes better than clone $\mathrm{C} 14$. Although the $P$. ramosa isolate/clone with the higher starting dose has a higher likelihood to succeed, its success ultimately depends on its competitiveness (Figure 4). Based on spore counts 20 days post-infection, it appears that the competitive outcome is largely decided during the first half of the parasite's growth phase (Figure 3).

Our results extend previous studies of multiple infections in the D. magna-P. ramosa host-parasite system, which were conducted using just parasite isolates $[19,27]$. First, we show for the first time that the effects of multiple infections by parasite clones could be different than those previously reported for isolates, because $P$. ramosa clones were less virulent yet produced more transmission stages than $P$. ramosa isolates. Second, we show that $P$. ramosa isolates/clones vary in their within-host competitiveness and ability to induce host castration. Third, we show that epidemiology (i.e., relative dose) affects the outcome of within-host competition (previous studies in this system used the same dose but in equal concentrations). Taken together, these results highlight the need to investigate multiple infections using a wider range of host and parasite genotypes and under diverse epidemiological scenarios.

Parasites that castrate their hosts are expected to inhibit host reproduction early in the infection process, in order to divert host resources for parasitic use [44-46]. The higher fecundity of $D$. magna singly infected with P1 in comparison with other isolates/clones suggests that some $P$. ramosa clones are more successful at inducing castration. If inducing castration bears a cost to the parasite, in the form of slowing down spore development 


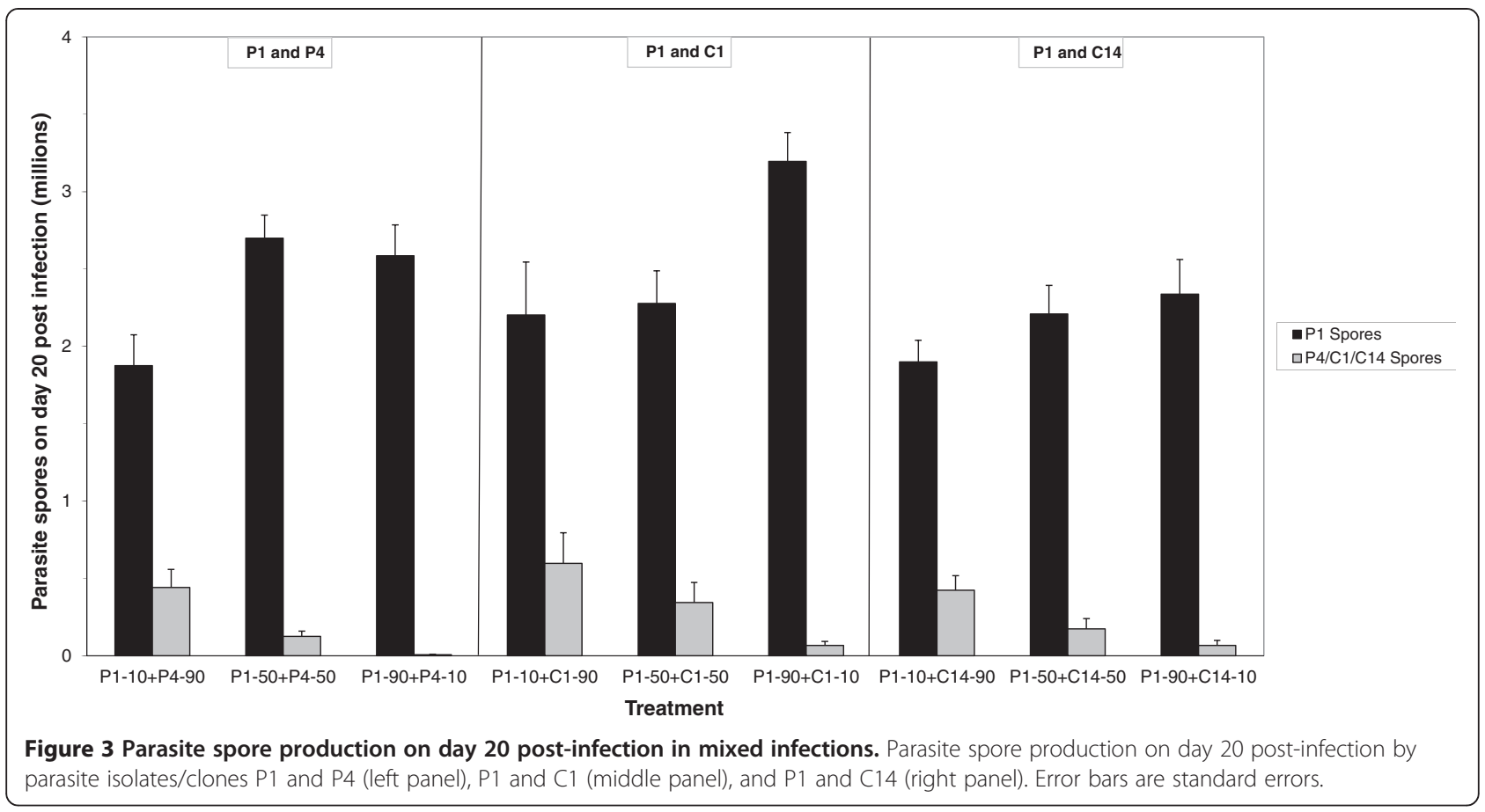

and growth, then it may affect its competitive ability with other clones. In mixed infections, the inability of P1 to castrate its host as quickly as P2 may be compensated by the greater competitiveness of P1. Put differently, castrating the host after it has reproduced once may be less costly to the parasite than doing so immediately after penetration, and may allow the parasite to focus on replicating itself to achieve a competitive edge [47]. P1 might also be benefitting if coinfecting isolates/clones in mixed infection induce castration [48]. It could be argued that $P$. ramosa sterilizes $D$. magna mechanistically, e.g., by growing around its ovaries. This is likely to bear no costs to the parasite, and may be supported by the fact that antibiotic treatment is sufficient to regain host reproduction [49]. However, our day 20 post-infection data suggest that P1 grows faster than its competitors, despite delaying castration. Furthermore, it is not unusual for infected D. magna to release a clutch after a long period of castration.

The transitive relationship in competitiveness in mixed infections with equal concentrations (i.e., spore production of $\mathrm{P} 4>\mathrm{C} 1>\mathrm{C} 14$ ) is in line with their relative virulence in single infections ( $\mathrm{P} 4$ was more virulent than $\mathrm{C} 1$ and $\mathrm{C} 14$ ). This suggests that when both parasite strains have equal chances to infect the host (50:50 concentration), their relative virulence in single infections may point to their competitive success in mixed infections. Similar results have been reported in a rodent malaria host-parasite system [24]. Our study extends these results by showing that even in unequal concentrations $\mathrm{P} 4$ produced more or at least as many spores as $\mathrm{C} 1$ and $\mathrm{C} 14$ during mixed infections with
P1/P2, despite its significantly lower spore throughput in single infections. Therefore, the ability of a parasite to transmit under conditions of frequent multiple infections ultimately depends on its competitiveness, and that a parasite's relative virulence (but not its replication rate) in single infections serves as a good indicator of its competitive ability. Moreover, if more virulent parasite strains are more often better competitors, frequent multiple infections will lead to higher levels of virulence [13].

Pasteuria ramosa clones have been found to exhibit strong GxG interactions for infectivity [43]. Some D. magna clones exhibit either complete resistance or complete susceptibility to infection that is governed by a simple genetic basis (i.e., one or few loci with dominance; [50]). Although the specificity of attachment to the host esophagus depends on both host and parasite genotypes [51], the specificity of $P$. ramosa proliferation within $D$. manga is poorly understood. It is also unknown whether the number of successful infections (i.e., number of spores attaching to the host esophagus) affects parasite replication rates within the host and the resulting spore load. Single-spore infection trials in the laboratory suggest that even though a single $P$. ramosa spore can cause disease, the likelihood of such an event is extremely low (circa 1 in 700 ; [43]). Spores that do not penetrate do not seem to be targeted by any innate immunity [52]. It might very well be that if $P$. ramosa spores penetrate the host in small numbers, they are cleared by the host's innate immune system before they are able to proliferate [53]. Direct interference or apparent competition among different P. ramosa clones may also reduce proliferation [54]. Since 


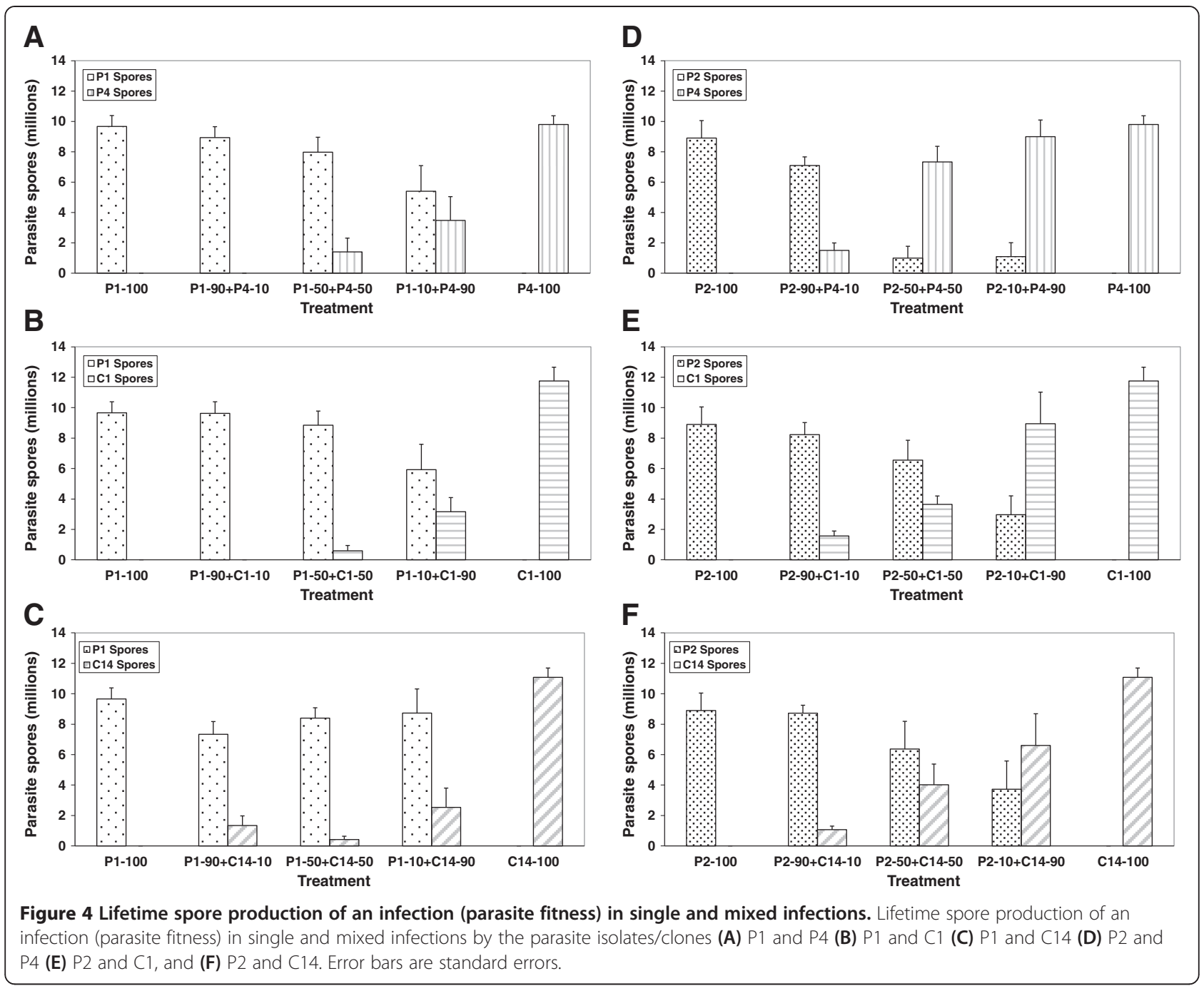

it is likely that $P$. ramosa isolates consist of more than one clone, some of which may be incompatible with the D. magna clone used in this experiment, we conjecture that a combination of proliferation specificity and interclone competition may explain why P. ramosa clones produced more spores than isolates. In other words, infection by a $P$. ramosa clone would maximize parasite fitness better than infection by a $P$. ramosa isolate. It remains to be determined whether the observed GxG interactions for infectivity also apply to within-host competitiveness and virulence (by examining the expression and evolution of virulence using additional $D$. magna clones).

Our finding that the competitive outcome is largely determined during the first half of the parasite's growth phase may be explained in several ways. First, the replication rates of successful competitors may be considerably higher than those of their counterparts, as evident from spore counts on day 20 post-infection. Second, direct interference or apparent competition might take place very early in the infection process e.g., [53], and clear out or considerably harm less competitive $P$. ramosa clones. Lastly, successful competitors might be able to facultatively upregulate their replication rates upon detection of another genotype within the same host, and thus express higher virulence [55-58]. To provide support to one or more of these conjectures would necessitate monitoring the competitive outcome during the initial growth phase while controlling for the number of successful infections.

Interestingly, the virulence of $P$. ramosa clones was lower than that of isolates. In theory under a scenario of resource competition, kin selection should reduce the increase in virulence per genotype in multiple infections by closely-related competing genotypes [59,60]. However, the relationship between virulence and relatedness depends on the social behavior displayed by the parasites, i.e., prudent exploitation, public goods cooperation or spite [14]. Evidence for reduced overall virulence in coinfections by closely-related parasite strains compared to unrelated strains is scarce $[15,16,61]$. Under the assumption that more than one $P$. ramosa spore penetrates the host during 
seven days of exposure, the present study provides additional support for the prediction that high relatedness selects for prudent exploitation and thus low virulence. This is because the difference in virulence between $P$. ramosa clones and isolates could be explained by $<100 \%$ relatedness of genotypes in isolate infections. This latter statement assumes that a $P$. ramosa isolate consists of more than one $P$. ramosa clone. It remains to be seen whether the increase in overall virulence under multiple infections with potentially unrelated genotypes resulted from increased host exploitation or the inability of the D. magna immune system to cope with antigenic diversity [17].

The dose levels used in the present study were chosen to achieve high infection rates (>90\%). Infection prevalence in natural populations of $D$. magna varies widely and may reach in certain ponds or years 100\% [62-65]. However, it is unknown whether naturally occurring $D$. magna populations are exposed to concentrations of $P$. ramosa spores similar to those administered in our experiment. Lower spore concentrations may decrease the likelihood of multiple infections, and thus alter both within- and betweenhost dynamics. For example, if multiple infections are rare, less virulent $P$. ramosa clones that produce more transmission stages may be selected over more virulent clones that are less infective and/or produce fewer transmission stages [19]. We do not expect different dose-response relationships for lower levels of infection, in terms of within-host competitiveness, overall virulence and parasite transmission. However, changes in the likelihood of multiple infections will affect the evolution of virulence.

\section{Conclusions}

The main finding of this study is that parasite isolates differ from parasite clones in their virulence and lifetime spore production of an infection. Moreover, parasite isolates/ clones differ in their within-host competitiveness and ability to induce host castration. Finally, the relative virulence and relative dose of coinfecting parasite strains strongly affect the competitive outcome. Taken together, our results emphasize the importance of epidemiology as well as of various parasite traits in determining the outcome of within-host competition. Incorporating realistic epidemiological and ecological conditions when testing theoretical models of multiple infections [66], as well as using a wider range of host and parasite genotypes, will enable us to better understand the course of virulence evolution.

\section{Methods}

\section{Biological system}

The host, Daphnia magna Straus, is a cyclical parthenogenetic crustacean parasitized by a wide variety of bacterial, microsporidial, oomycetes and fungal parasites $[67,68]$. The parasite, Pasteuria ramosa Metchnikoff 1888, is an endospore-forming, gram-positive bacterium of Daphnia with strict horizontal transmission, in which infective stages (i.e., spores) are released from the decaying cadaver of the host $[67,69]$. It castrates and severely reduces the survival of the host, which rarely produces any offspring after infection [44]. Infections are clearly visible two weeks post-infection, because infected animals have a brownishreddish color and do not carry eggs. In field populations of D. magna, many parasites may coexist in the same pond and multiple infections of host individuals by several $P$. ramosa strains $[70,71]$ or different parasite species [72-74] are often observed.

\section{Host and parasite collections}

We used a single D. magna clone ( $\mathrm{HO} 2)$ originally collected from a pond in Hungary, by isolating parthenogenetic eggs from the brood chamber of an uninfected adult female and raising the clonal offspring in isolation under standardized laboratory conditions. In preparation to the experiment we stock-cultured D. magna in 400-mL glass beakers, each containing eight individuals with artificial medium [75,76], where they were fed daily with $1.5 \times 10^{5}$ cells $\mathrm{mL}^{-1}$ medium of the chemostat-cultured unicellular algae Scenedesmus gracilis.

The three $P$. ramosa isolates used in this experiment were obtained either from one infected D. magna female (P1: Gaarzerfeld, Germany, 1997; P2: Kains, England, 2002), or from several infected D. magna individuals (P4: Heverlee, Belgium, 2003). Isolates are a naturally occurring feature of the Daphnia-Pasteuria host-parasite system. As such, they are relevant to evolutionary processes in natural populations. These isolates had been used in the laboratory in the past 15 years, and all of them were propagated through the experimental host clone $\mathrm{HO} 2$, to obtain enough sporecarrying cadavers to produce sufficient amounts of spore suspensions for the experiment. Two of these isolates (P1 and P4) were also used in a previous study of multiple infections of D. magna [19]. The use of laboratorymaintained lines is not unusual in many experimental hostparasite model systems and can be justified by the use of these lines to test mechanistic hypotheses, as we do in our study. The $P$. ramosa clones $\mathrm{C} 1$ and $\mathrm{C} 14$ were obtained respectively from isolate P5 (Moscow, Russia, 1996) and isolate P3 (Tvärminne, Finland, 2002) via infection by limited dilution (technical details in [43]). These P. ramosa clones were also propagated through the experimental host clone $\mathrm{HO} 2$. All cadavers were carefully homogenized and spore concentrations were determined using a Thoma counting chamber (depth: $0.02 \mathrm{~mm}$, square width: $0.05 \mathrm{~mm}$ ).

\section{Experimental design and setup}

We followed a cohort of 1,344 D. magna individuals and examined the outcome of single and mixed infections. In total there were 39 treatments, each with 28 replicates, as depicted in Table 3: 20 single infection treatments (three $P$. 
ramosa isolates and two P. ramosa clones, each using four dose levels), 18 mixed infection treatments (combinations of either $\mathrm{P} 1$ or $\mathrm{P} 2$ with one of $\mathrm{P} 4, \mathrm{C} 1$ and $\mathrm{C} 14$, using spore mixtures of 90,000:10,000, 50,000:50,000 and 10,000:90,000 spores), as well as an unexposed control group. In nine of the 18 mixed infection treatments (those with P1), we doubled the number of replicates and used the extra 28 replicates to examine parasite spore production and the competitive outcome on day 20 post-infection. Throughout the experiment and on a daily basis, we monitored $D$. magna survival, release of offspring and the amount of $P$. ramosa spores following the host's death. We defined virulence as time-to-host-death-since-exposure (i.e., host longevity). We chose this definition, instead of "reduction in host fitness following infection", because the trade-off model is based on "parasite-induced host mortality" being the right definition for the virulence of horizontally-transmitted parasites [77]. Model predictions using expected host longevity may differ from those obtained using host mortality rate [78], yet in our one-generation study these effects are most likely negligible. Host fitness was defined as the lifetime number of offspring produced. Parasite fitness was estimated from the number of spores at the time of host death, which is equal to the lifetime spore production of an infection.

We used offspring of the third generation of the $\mathrm{HO} 2$ isofemale line to minimize maternal effects. To start the experiment we separated newborns from the $D$. magna clone line (0-24 h old) into four 400-mL beakers and fed them daily with $1.5 \times 10^{5}$ algae cells $\mathrm{mL}^{-1}$ medium. On day four we placed single females of D. magna in $100-\mathrm{mL}$ jars, filled with $20 \mathrm{~mL}$ of artificial medium, and initially fed them $2 \times 10^{6}$ algae cells per animal per day. The infection treatment was performed on day five. A week later, on day 12, we replaced the medium of all animals with $100 \mathrm{~mL}$ of fresh medium and thereafter medium was replaced on a weekly basis. To accommodate the growing food demands of the growing animals, on days 9, 15, 18, $22,27,30$ and 37 we increased the daily food level for all individuals to $3 \times 10^{6}, 5 \times 10^{6}, 6 \times 10^{6}, 7 \times 10^{6}, 8 \times 10^{6}, 9 \times$ $10^{6}$ and $10 \times 10^{6}$ algae cells per day, respectively.

The temperature was $20 \pm 0.5^{\circ} \mathrm{C}$ and the light:dark cycle was $16 \mathrm{~h}: 8 \mathrm{~h}$. All treatments were randomly distributed across the shelves of two incubators and their position was rearranged frequently to avoid position effects. Offspring counts and dead animals were recorded daily. Animals that had died after day 16 (since birth) were dissected and checked for disease using phase contrast microscopy (300-600x). Animals that had died earlier could not be reliably scored for infection and were thus excluded from the analyses. The experiment was terminated after all animals had died. The dead D. magna were frozen in $0.1 \mathrm{~mL}$ of medium at $-20^{\circ} \mathrm{C}$ for subsequent parasite spore counting with a haemocytometer.

\section{Genetic analyses}

To trace the relative success of $P$. ramosa isolates and clones during mixed infections, we used variable number of tandem repeats (VNTR) markers. We used the previously developed primers Pr1, Pr2 and Pr3 (for details, see [70] and Table 2 in [19]). These primers allow distinguishing between $P$. ramosa isolates $\mathrm{P} 1$ and $\mathrm{P} 3 / \mathrm{P} 4 / \mathrm{P} 5$, and between isolates $\mathrm{P} 2$ and $\mathrm{P} 3 / \mathrm{P} 4 / \mathrm{P} 5$. Because $P$. ramosa clones $\mathrm{C} 1$ and $\mathrm{C} 14$ were derived from isolates $\mathrm{P} 5$ and P3, respectively, the same primers can be used to distinguish between isolate $\mathrm{P} 1$ and clones $\mathrm{C} 1 / \mathrm{C} 14$, and between

Table 3 Overview of the treatments in the experiment

\begin{tabular}{|c|c|c|c|}
\hline Treatment & Type of infection & Infection dose & Abbreviations of combinations used \\
\hline PX-10 & Single & 10,000 spores of $\mathrm{PX}$ & P1-10, P2-10, P4-10, C1-10, C14-10 \\
\hline PX-50 & Single & 50,000 spores of $\mathrm{PX}$ & P1-50, P2-50, P4-50, C1-50, C14-50 \\
\hline PX-90 & Single & 90,000 spores of $\mathrm{PX}$ & P1-90, P2-90, P4-90, C1-90, C14-90 \\
\hline PX-100 & Single & 100,000 spores of $\mathrm{PX}$ & P1-100, P2-100, P4-100, C1-100, C14-100 \\
\hline \multirow[t]{3}{*}{$P X-10+P Y-90$} & Mixed & 10,000 spores of $\mathrm{PX}$ and 90,000 spores of $\mathrm{PY}$ & P1-10 + P4-90, P1-10 + C1-90, \\
\hline & & & P1-10 + C14-90, P2-10 + P4-90, \\
\hline & & & $\mathrm{P} 2-10+\mathrm{C} 1-90, \mathrm{P} 2-10+\mathrm{C} 14-90$ \\
\hline \multirow[t]{3}{*}{$P X-50+P Y-50$} & Mixed & 50,000 spores of $P X$ and 50,000 spores of $P Y$ & $\mathrm{P} 1-50+\mathrm{P} 4-50, \mathrm{P} 1-50+\mathrm{C} 1-50$ \\
\hline & & & $\mathrm{P} 1-50+\mathrm{C} 14-50, \mathrm{P} 2-50+\mathrm{P} 4-50$ \\
\hline & & & $\mathrm{P} 2-50+\mathrm{C} 1-50, \mathrm{P} 2-50+\mathrm{C} 14-50$ \\
\hline \multirow[t]{3}{*}{$P X-90+P Y-10$} & Mixed & 90,000 spores of $P X$ and 10,000 spores of PY & P1-90 + P4-10, P1-90 + C1-10, \\
\hline & & & P1-90 + C14-10, P2-90 + P4-10, \\
\hline & & & $P 2-90+C 1-10$, P2-90 + C14-10 \\
\hline Control & None & None & Control \\
\hline
\end{tabular}

In single infections, PX stands for either one of the three $P$. ramosa isolates $\mathrm{P} 1, \mathrm{P} 2$ and $\mathrm{P} 4$, or one of the two $\mathrm{P}$. ramosa clones $\mathrm{C} 1$ and $\mathrm{C} 14$. In mixed infections, $\mathrm{PX}$ stands for either $\mathrm{P} 1$ or $\mathrm{P} 2$, and $\mathrm{PY}$ stands for one of $\mathrm{P} 4, \mathrm{C} 1$ and $\mathrm{C} 14$. 
isolate $\mathrm{P} 2$ and clones $\mathrm{C} 1 / \mathrm{C} 14$. The protocol employed resembles the one used by [19] with the following changes. Spore solutions were suspended in $300 \mu \mathrm{L}$ water and $30 \mu \mathrm{L}$ proteinase $\mathrm{K}(20 \mathrm{mg} / \mathrm{mL})$. We added approximately $160 \mathrm{mg}$ of $0.1 \mathrm{~mm}$ zirconia beads, subjected them to beating for $20 \mathrm{~s}$ at full speed, and incubated them at $56^{\circ} \mathrm{C}$ for $30 \mathrm{~min}$. We then spinned down beads at $5000 \mathrm{~g}$ for $30 \mathrm{~s}$, transferred supernatant to a new tube and continued with peqGOLD Tissue DNA Mini Kit (Peqlab, Erlangen, Germany). The relative intensity of the peaks of the $P$. ramosa isolate/clone specific microsatellite markers were analyzed with AB3130xl Sequencer (Applied Biosystems, Foster City, USA) and interpreted as the relative proportion of spores of different $P$. ramosa isolates/clones in an individual D. magna as described in [19]. Spore counts for each $P$. ramosa isolate/clone in mixed infections were derived by multiplying the abovementioned relative proportion by the total number of spores produced following infection.

\section{Statistical analyses}

All statistical tests were done using SPSS for Windows release 19.0.0.1 (SPSS Inc. 2010). The effects on virulence, host and parasite fitness were investigated using general linear models (GLM). Because time-to-host-death was normally distributed and the experiment ended only after all hosts had died, there was no need for censoring data and using specific survival analysis procedures. When necessary, parasite spore production and host offspring counts were square-root-transformed to meet the normality and homoscedasticity assumptions. In GLM procedures dose level and parasite isolate/clone were considered fixed factors. Dose level was only used to compare single infection treatments with spore mixtures of 10,000, 50,000, 90,000 and 100,000 spores (see Table 2). The dichotomous variable parasite isolate/clone was used to compare the virulence and spore production of $P$. ramosa isolates vs. clones. Thereafter, contrasts were used to test specific hypotheses in subsets of the total dataset.

\section{Competing interests}

The authors declare that they have no competing interests.

\section{Authors' contributions}

FBA conceived and designed the study, carried out the infection assays, performed the statistical analysis and drafted the manuscript. JR carried out the molecular genetic work and helped to draft the manuscript. Both authors read and approved the final manuscript.

\section{Acknowledgements}

We thank S. Alizon, M. Brockhurst, D. Ebert and four anonymous reviewers for helpful comments on this manuscript.

Received: 19 December 2012 Accepted: 29 April 2013

Published: 3 May 2013

\section{References}

1. Read AF, Taylor LH: The ecology of genetically diverse infections. Science 2001, 292:1099-1102.
2. Rigaud T, Perrot-Minnot M-J, Brown MJF: Parasite and host assemblages: embracing the reality will improve our knowledge of parasite transmission and virulence. Proc R SOC B 2010, 277:3693-3702.

3. López-Villavicencio M, Jonot O, Coantic A, Hood ME, Enjalbert J, Giraud T: Multiple infections by the anther smut pathogen are frequent and involve related strains. PLoS Pathog 2007, 3:e176.

4. Vardo-Zalik AM: Clonal diversity of a malaria parasite, Plasmodium mexicanum, and its transmission success from its vertebrate to insect host. Int J Parasitol 2009, 39:1573-1579.

5. Clavijo G, Williams T, Muñoz D, Caballero P, López-Ferber M: Mixed genotype transmission bodies and virions contribute to the maintenance of diversity in an insect virus. Proc R Soc B 2010, 277:943-951.

6. Taerum SJ, Cafaro MJ, Currie CR: Presence of multiparasite infections within individual colonies of leaf-cutter ants. Environ Entomol 2010, 39:105-113

7. Balmer $\mathrm{O}$, Tanner M: Prevalence and implications of multiple-strain infections. Lancet Infect Dis 2011, 11:868-878.

8. Gandon S, Day T: Evidences of parasite evolution after vaccination. Vaccine 2008, 26:C4-C7.

9. Mostowy R, Salathé M, Kouyos RD, Bonhoeffer S: On the evolution of sexual reproduction in hosts coevolving with multiple parasites. Evolution 2010, 64:1644-1656.

10. Frank SA: Models of parasite virulence. Q Rev Biol 1996, 71:37-78.

11. May RM, Nowak MA: Coinfection and the evolution of parasite virulence. Proc R Soc B 1995, 261:209-215.

12. Mosquera J, Adler FR: Evolution of virulence: a unified framework for coinfection and superinfection. J Evol Biol 1998, 195:293-313.

13. van Baalen M, Sabelis MW: The dynamics of multiple infection and the evolution of virulence. Am Nat 1995, 146:881-910

14. Buckling A, Brockhurst MA: Kin selection and the evolution of virulence. Heredity 2008, 100:484-488.

15. Brown SP, Hochberg ME, Grenfell BT: Does multiple infection select for raised virulence? Trends Microbiol 2002, 10:401-405.

16. López-Villavicencio M, Courjol F, Gibson AK, Hood ME, Jonot O, Shykoff JA, Giraud T: Competition, cooperation among kin, and virulence in multiple infections. Evolution 2011, 65:1357-1366.

17. Davies $C M$, Fairbrother $E$, Webster JP: Mixed strain schistosome infections of snails and the evolution of parasite virulence. Parasitology 2002, 124:31-38.

18. Taylor LH, Mackinnon MJ, Read AF: Virulence of mixed-clone and singleclone infections of the rodent malaria Plasmodium chabaudi. Evolution 1998, 52:583-591.

19. Ben-Ami F, Mouton L, Ebert D: The effects of multiple infections on the expression and evolution of virulence in a Daphnia-endoparasite system. Evolution 2008, 62:1700-1711.

20. Hughes WOH, Petersen KS, Ugelvig LV, Pedersen D, Thomsen L, Poulsen M, Boomsma JJ: Density-dependence and within-host competition in a semelparous parasite of leaf-cutting ants. BMC Evol Biol 2004, 4:45.

21. Wargo AR, Garver KA, Kurath $G$ : Virulence correlates with fitness in vivo for two M group genotypes of Infectious hematopoietic necrosis virus (IHNV). Virology 2010, 404:51-58.

22. Bashey F, Reynolds C, Sarin T, Young SK: Virulence and competitive ability in an obligately killing parasite. Oikos 2011, 120:1539-1545.

23. Alizon S, de Roode JC, Michalakis Y: Multiple infections and the evolution of virulence. Ecol Lett 2013, 16:556-567.

24. Bell AS, de Roode JC, Sim D, Read AF: Within-host competition in genetically diverse malaria infections: parasite virulence and competitive success. Evolution 2006, 60:1358-1371.

25. Hood ME: Dynamics of multiple infection and within-host competition by the Anther-Smut pathogen. Am Nat 2003, 162:122-133.

26. Jäger I, Schjørring S: Multiple infections: relatedness and time between infections affect the establishment and growth of the cestode Schistocephalus solidus in its stickleback host. Evolution 2006, 60:616-622.

27. Ben-Ami F, Rigaud T, Ebert D: The expression of virulence during double infections by different parasites with conflicting host exploitation and transmission strategies. J Evol Biol 2011, 24:1307-1316.

28. Lohr JN, Yin M, Wolinska J: Prior residency does not always pay off co-infections in Daphnia. Parasitology 2010, 137:1493-1500. 
29. Gower CM, Webster JP: Intraspecific competition and the evolution of virulence in a parasitic trematode. Evolution 2005, 59:544-553.

30. Fellous S, Koella JC: Infectious dose affects the outcome of the withinhost competition between parasites. Am Nat 2009, 173:E177-E184.

31. Ben-Ami F, Regoes RR, Ebert D: A quantitative test of the relationship between parasite dose and infection probability across different hostparasite combinations. Proc R Soc B 2008, 275:853-859.

32. Regoes RR, Hottinger JW, Sygnarski L, Ebert D: The infection rate of Daphnia magna by Pasteuria ramosa conforms with the mass-action principle. Epidemiol Infect 2003, 131:957-966.

33. Ben-Ami F, Ebert D, Regoes RR: Pathogen dose infectivity curves as a method to analyze the distribution of host susceptibility: a quantitative assessment of maternal effects after food stress and pathogen exposure. Am Nat 2010, 175:106-115.

34. van der Werf W, Hemerik L, Vlak JM, Zwart MP: Heterogeneous host susceptibility enhances prevalence of mixed-genotype micro-parasite Infections. PLoS Comput Biol 2011, 7:e1002097.

35. de Roode JC, Gold LR, Altizer S: Virulence determinants in a natural butterfly-parasite system. Parasitology 2007, 133:657-668.

36. Perlman SJ, Jaenike J: Competitive interactions and persistence of two nematode species that parasitize Drosophila recens. Ecol Lett 2001, 4:577-584.

37. Jensen KH, Little TJ, Skorping A, Ebert D: Empirical support for optimal virulence in a castrating parasite. PLoS Biol 2006, 4:1265-1269.

38. de Roode JC, Yates AJ, Altizer S: Virulence-transmission trade-offs and population divergence in virulence in a naturally occurring butterfly parasite. Proc Natl Acad Sci U S A 2008, 105:7489-7494

39. Froissart R, Doumayrou J, Vuillaume F, Alizon S, Michalakis Y: The virulencetransmission trade-off in vector-borne plant viruses: a review of (non-) existing studies. Phil Trans R Soc B 2010, 365:1907-1918.

40. Regoes RR, Nowak MA, Bonhoeffer S: Evolution of virulence in a heterogeneous host population. Evolution 2000, 54:64-71.

41. Kirchner JW, Roy BA: Evolutionary implications of host-pathogen specificity: fitness consequences of pathogen virulence traits. Evol Ecol Res 2002, 4:27-48.

42. Garamszegi LZ: The evolution of virulence and host specialization in malaria parasites of primates. Ecol Lett 2006, 9:933-940.

43. Luijckx P, Ben-Ami F, Mouton L, Du Pasquier L, Ebert D: Cloning of the unculturable parasite Pasteuria ramosa and its Daphnia host reveals extreme genotype-genotype interactions. Ecol Lett 2011, 14:125-131.

44. Ebert D, Carius HJ, Little TJ, Decaestecker E: The evolution of virulence when parasites cause host castration and gigantism. Am Nat 2004, 164:S19-S32.

45. O'Keefe KJ, Antonovics J: Playing by different rules: the evolution of virulence in sterilizing pathogens. Am Nat 2002, 159:597-605.

46. Obrebski S: Parasite reproductive strategy and evolution of castration of hosts by parasites. Science 1975, 188:1314-1316.

47. Day T: Virulence evolution and the timing of disease life-history events. Trends Ecol Evol 2003, 18:113-118.

48. Hughes $\mathrm{WOH}$, Boomsma JJ: Let your enemy do the work: within-host interactions between two fungal parasites of leaf-cutting ants. Proc $R$ Soc B (Suppl) 2004, 271:S104-S106.

49. Little TJ, Ebert D: The cause of parasitic infection in natural populations of Daphnia (Crustacea: Cladocera): the role of host genetics. Proc R Soc B 2000, 267:2037-2042.

50. LuijckX P, Fienberg H, Duneau D, Ebert D: Resistance to a bacterial parasite in the crustacean Daphnia magna shows Mendelian segregation with dominance. Heredity 2012, 108:547-551.

51. Duneau D, Luijckx P, Ben-Ami F, Laforsch C, Ebert D: Resolving the infection process reveals striking differences in the contribution of environment, genetics and phylogeny to host-parasite interactions. BMC Biol 2011, 9:11

52. King KC, Auld SKJR, Wilson PJ, James J, Little TJ: The bacterial parasite Pasteuria ramosa is not killed if it fails to infect: implications for coevolution. Ecol Evol 2013, 3:197-203.

53. Auld SKJR, Scholefield JA, Little TJ: Genetic variation in the cellular response of Daphnia magna (Crustacea: Cladocera) to its bacterial parasite. Proc R SOC B 2010, 277:3291-3297.

54. Massey RC, Buckling A, ffrench-Constant R: Interference competition and parasite virulence. Proc R Soc B 2004, 271:785-788

55. Kümmerli R, Jiricny N, Clarke LS, West SA, Griffin AS: Phenotypic plasticity of a cooperative behaviour in bacteria. J Evol Biol 2009, 22:589-598.
56. Pollitt LC, Mideo N, Drew DR, Schneider P, Colegrave N, Reece SE: Competition and the evolution of reproductive restraint in malaria parasites. Am Nat 2011, 177:358-367.

57. Reece SE, Ramiro RS, Nussey DH: Plastic parasites: sophisticated strategies for survival and reproduction? Evol Appl 2009, 2:11-23.

58. Reece SE, Drew DR, Gardner A: Sex ratio adjustment and kin discrimination in malaria parasites. Nature 2008, 453:609-614.

59. Chao L, Hanley KA, Burch CL, Dahlberg C, Turner PE: Kin selection and parasite evolution: higher and lower virulence with hard and soft selection. Q Rev Biol 2000, 75:261-275.

60. Frank SA: A kin selection model for the evolution of virulence. Proc $R$ Soc B 1992, 250:195-197.

61. West SA, Buckling A: Cooperation, virulence and siderophore production in bacterial parasites. Proc R Soc B 2003, 270:37-44.

62. Duncan $A B$, Little TJ: Parasite-driven genetic change in a natural population of Daphnia. Evolution 2007, 61:796-803.

63. Duncan AB, Mitchell SE, Little TJ: Parasite-mediated selection and the role of sex and diapause in Daphnia. J Evol Biol 2006, 19:1183-1189.

64. Little TJ, Ebert D: Associations between parasitism and host genotype in natural populations of Daphnia (Crustacea: Cladocera). J Anim Ecol 1999, 68:134-149.

65. Mitchell SE, Read AF, Little TJ: The effect of a pathogen epidemic on the genetic structure and reproductive strategy of the crustacean Daphnia magna. Ecol Lett 2004, 7:848-858.

66. Asplen MK, Bruns E, David AS, Denison RF, Epstein B, Kaiser MC, Kaser JM, Lacroix C, Mohl EK, Quiram G, et al: Do trade-offs have explanatory power for the evolution of organismal interactions? Evolution 2012, 66:1297-1307.

67. Ebert D: Ecology, Epidemiology, and Evolution of Parasitism in Daphnia [Internet]. Bethesda (MD): National Library of Medicine (US), National Center for Biotechnology Information; 2005. Available from: http://www.ncbi.nlm. nih.gov/books/NBK2036/.

68. Green J: Parasites and epibionts of Cladocera. Trans Zool Soc Lond 1974, 32:417-515.

69. Ebert D, Rainey P, Embley TM, Scholz D: Development, life cycle, ultrastructure and phylogenetic position of Pasteuria ramosa Metchnikoff 1888: rediscovery of an obligate endoparasite of Daphnia magna Straus. Phil Trans R Soc B 1996, 351:1689-1701.

70. Mouton L, Nong G, Preston JF, Ebert D: Variable-number tandem repeats as molecular markers for biotypes of Pasteuria ramosa in Daphnia spp. Appl Environ Microbiol 2007, 73:3715-3718.

71. Andras JP, Ebert D: A novel approach to parasite population genetics: experimental infection reveals geographic differentiation, recombination and host-mediated population structure in Pasteuria ramosa, a bacterial parasite of Daphnia. Mol Ecol 2013, 22:972-986.

72. Decaestecker E, Declerck S, De Meester L, Ebert D: Ecological implications of parasites in natural Daphnia populations. Oecologia 2005, 144:382-390.

73. Stirnadel HA, Ebert D: Prevalence, host specificity and impact on host fecundity of microparasites and epibionts in three sympatric Daphnia species. J Anim Ecol 1997, 66:212-222

74. Goren L, Ben-Ami F: Ecological correlates between cladocerans and their endoparasites from permanent and rain pools: patterns in community composition and diversity. Hydrobiologia 2013, 701:13-23.

75. Ebert D, Zschokke-Rohringer CD, Carius HJ: Within- and betweenpopulation variation for resistance of Daphnia magna to the bacterial endoparasite Pasteuria ramosa. Proc R Soc B 1998, 265:2127-2134.

76. Klüttgen $B$, Dümler U, Engels M, Ratte HT: ADaM, an artificial freshwater for the culture of zooplankton. Water Res 1994, 28:743-746.

77. Alizon S, Hurford A, Mideo N, van Baalen M: Virulence evolution and the trade-off hypothesis: history, current state of affairs and the future. J Evol Biol 2009, 22:245-259.

78. Day T: On the evolution of virulence and the relationship between various measures of mortality. Proc R Soc B 2002, 269:1317-1323.

doi:10.1186/1471-2148-13-97

Cite this article as: Ben-Ami and Routtu: The expression and evolution of virulence in multiple infections: the role of specificity, relative virulence and relative dose. BMC Evolutionary Biology 2013 13:97. 